\title{
Optimization of Image Segmentation Method Based on Improved Wavelet Algorithm
}

\author{
Sun li \\ Luzhou Vocational \& Technical College, Luzhou, Sichuan, 646005 China \\ 307454075@qq.com
}

Keywords: image segmentation; wavelet transform; multi scale

\begin{abstract}
In the optimization image segmentation process, the traditional algorithm is used for segmentation, because the calculation method is complex, image segmentation accuracy rate is low, the clarity is not high, when the amount of information in the image segmentation is very large, the time consumption is big. Aiming at the above problems, an improved image optimization segmentation method is proposed based on wavelet algorithm. The image feature is obtained based on wavelet transform, the image characteristics can be reacted in local information, the advantage is obvious, the adjacent scale wavelet coefficients multiplication method isused to remove the noise, and the wavelet algorithm and graph theory algorithm is fused to generate the minimum spanning tree, the accurate image segmentation is obtained with minimum spanning tree. The simulations show the method is applied in image segmentation, the precision is high, and it has strong applicability.
\end{abstract}

\section{Introduction}

In the research field of image, the technology of image segmentation is a kind of very important technology of image processing, it is widely applied in many aspects of image processing, the general idea of image segmentation technology is to put a large image to divideinto many small pieces, previous research shows the methods and types of image segmentation is great many. But there are no unified standard to measure, this has brought trouble tothe large-scale application. So how to establish a reasonable optimization of general image segmentation method is the key to solve the issue, more and more experts have put a lot ofrelated attention to the field. At this stage, the main methods of image segmentation include ant colony algorithm, image segmentation method based on pixel gray difference algorithm, and image segmentation method based on adaptive threshold segmentation algorithm. Among them, the most commonly used method is the image segmentation based on adaptive thresholdsegmentation algorithm. According to the traditional image segmentation methods, the image surface is core, this method is used in segmentation, it has strong operability, it is simple and it has obtained the broad application. But its disadvantage is that the surface is often leads to excessive segmentation, the segmentation performance is bad. The computational complexity is big[1].

Therefore, an improved image optimization segmentation method is proposed based on wavelet algorithm. The image feature is obtained based on wavelet transform, the image characteristics can be reacted in local information, the advantage is obvious, the adjacent scale wavelet coefficients multiplication method is used to remove the noise, and the wavelet algorithm and graph theory algorithm is fused to generate the minimum spanning tree, the accurate image segmentation is obtained with minimum spanning tree. The simulations show the method is applied in image segmentation, the precision is high, and it has strong applicability.

\section{Image segmentation method based on improved wavelet algorithm}

A Image feature extraction and denoising.The advantage of wavelet transform is that it can provide the time domain and frequency domain window with the image features. It has the ability of reaction of the local features of image information, so it can consider to extract local features of images by using the characteristics of wavelet transform, wavelet transform is used for image 
processing, according to the first object of study by wavelet analysis, the wavelet analysis itself has no deformation[2], the wavelet analysis and moment invariants are combined, and the wavelet moment invariant is used to mine the details of the image control ability, in order to obtain the rotation invariant moment, the formula is adopt as:

For the continuous grey function $f(x, y)$, the continuous gray function is particularly effective to the moment rotation and the details of the image processing, its $(p+q)$ order two dimension origin moment $M_{p q}$ is defined as:

$$
\begin{aligned}
& M_{p q}=\int_{+\infty}^{-\infty} \int_{+\infty}^{-\infty} x^{p} y^{q} f(x, y) d x d y \\
& p, q=0,1,2, \ldots
\end{aligned}
$$

In the formula, $M_{p q}$ is the two-dimensional origin moment of continuous gray function, $p, q$ is the corresponding order, according to the uniqueness theorem[3], its order class is existent and unique, it can be described as $f(x, y)$, in turn, $f(x, y)$ can be determined by $\mathrm{t}$ all moments uniquely. Due to lack of shift invariance for $M_{p q}$, the $(p+q)$ order central moment is:

$$
\eta_{p q}=\frac{u^{p q}}{u^{r}}
$$

Where, $\eta_{p q}$ is the $(p+q)$ order central moment of $M_{p q}, p, q$ indicate the number of the corresponding order, $u$ is stage moment. $r=(p+q+2) / 2, p+q=2,3$, the invariant performance of image translation, rotation and scaling can be obtained based on the two order and three order normalized central moment[4].

According to the above content, the invariant wavelet can be defined as:

$$
\left\|\begin{array}{r}
\text { wave } \\
m, n
\end{array}\right\|=\left\|\int s_{q}(r) \varphi_{m, n}(r) r d r\right\|
$$

Where, ${ }^{\varphi_{m, n}}(r)=2^{\frac{m}{2}} \psi\left(2^{m} r-n\right)$, factually, $F_{m, n}^{\text {wavelet }}$ is the wavelet transform of $S_{q}(r) r$, because $\varphi_{m, n}(r)$ is compactly supported in general, each $F_{m, n}^{\text {wavelet }}$ is a detail description of the image, the image moments are calculated, and the error brought by noise will reduced, the error is much less than computing the whole image noise, generally use the three order B spline function as the mother wavelet[5], the formula is shown as follows:

$$
\psi(r)=\frac{4 a^{n+1}}{\sqrt{2 \pi(n+1)}} \sigma \omega \cos |2 \pi f o(2 r-1)| \times \exp \left(-\frac{(2 r-1)^{2}}{2 \sigma_{\omega}^{2}(n+1)}\right)
$$

Where, $n=3, \alpha=0.697066, f_{0}=0.49177, \sigma_{\sigma}^{2}=0.561145$. The image is processed with normalization, and the following results can be obtained:

In the range of $r \leq 1$, the different sizes factor $m$ is used, and the displacement factor $n, \varphi_{m, n}(r)$ is defined, the $n, \varphi_{m, n}(r)$ can traverse the entire radial space $[0,1]$, the wavelet moment $F_{m, n}^{\text {wavelet }}$ provides image features on different scales of $f(r, \theta)$ image, from the above analysis, we can get the characteristic information of image segmentation, including the global feature information and local feature information.

In the segmentation process of image, 98\% images have noise information, if you want to filter the noise, first we need to carry on the analysis of the image, from the study we find that the is a large part of image segmentation obey Gauss distribution. In the noise elimination, because only a 
few coefficients of wavelet signal exists in the domain, and the noise distribution in all the coefficients of wavelet transform, now we introduce the wavelet transform, the local rules of wavelet transform is detected, and the noise is filtered, the singular index is used to indicate the processing.

Assumed $(f, x) \in l^{2}(R)$, if $\forall x \in \delta x_{o}$, then:

$$
\left|(f x)-\left(f x_{0}\right)\right|=c\left|x-x_{0}\right|^{a}
$$

Where, singular index of $(f, x)$ at $x_{0}$ point can be described by $\partial$, the function ordinary degree increases with the value increasing, $C$ is the constant. Analysis showed that, the impulse function has the negative singularity exponent, the Gauss white noise has singular stochastic distribution function with everywhere singular performance, negative singular index is $\partial=-1 / 2-\xi, \forall \xi \succ 0$, the singular index of signal $\partial\rangle 0$, according to the positive and negative of $\partial$, the signal noise is eliminated.

B Optimization of image segmentation.The wavelet algorithm and graph algorithm are combined, the topological sort acyclic graph $G$ combines the minimum spanning tree, and the distribution characteristics of the image information are distributed in the different regions, the minimum spanning tree method is introduced, and the image segmentation is realized, and finally, the two value method is used for image sharpening.

Each region after the initial segmentation is treated as a node of undirected graph $G$, similarity between two nodes can be described by weight function, finally, the classification is taken according to the image property.

Undirected graph $G=[x, E]$ is a network with $N$ nodes, assumed $u$ is the set of vertices in spanning tree, minimum spanning tree is obtained based on wavelet algorithm and graph theory algorithm, the steps are shown as follows:

(1) $X_{0}$ is put in the set $u$;

(2) Find the edge with minimum weight, add to the spanning tree, where, the edge can be expressed as $u \in U, x \in X-U,(u, x) \in E$.

(3) Find the edge $x$, the edge is added to the set $u$, repeat second steps, until the set $u$ contains $n$ nodes.

Determine the data set of image, a minimum spanning tree is obtained, in the minimum spanning tree, the whose edge weights represent similarity degree between data samples, according to minimum spanning tree and the maximum between class distance, quality of an image are evaluated, an objective function is used for classification, the target function $\mathrm{Ht}$ is defined as follows:

$$
H t=\frac{\sum_{i=1}^{m} p_{i}\left(m_{i}-m\right)^{2}}{\sum_{i=1}^{m} \sum_{j=1}^{t}\left(x_{j}-m_{j}\right)^{2} h_{j}}
$$

Wherein, $\sum_{i=1}^{m} p_{i}\left(m_{i}-m\right)^{2}$ is the distance between classes, $\sum_{i=1}^{m} \sum_{j=1}^{t}\left(x_{j}-m_{j}\right)^{2} h_{j}$ is the distance between types, $m$ is the mean probability of image pixels, $p^{p_{i}}$ is the data samples appear probability of the number of clustering, $m_{j}$ is the mean value of $i$ th classification, $i$ is the number of clustering.

\section{Simulation and analysis}

In order to verify the performance of this algorithm, the simulation is taken, and the new algorithm is applied in image segmentation, the simulation platform is expressed as: $2 \mathrm{G}$ microcomputer, matlab 7.1 virtual platform, the experiment is simulated, and the image segmentation is realized, 
the traditional algorithm for image segmentation and the traditional algorithm for image segmentation are compared, the number of iterations is 99, 10 experiments are taken with the traditional algorithm and new algorithm, the experimental results are obtained,and shown in table 1.

Table 1 Image segmentation results

\begin{tabular}{|c|c|c|}
\hline $\begin{array}{l}\text { Number of } \\
\text { experiments } \\
\text { (time) }\end{array}$ & $\begin{array}{l}\text { Segmentation } \\
\text { accuracy of new } \\
\text { algorithm (\%) }\end{array}$ & $\begin{array}{l}\text { Segmentation } \\
\text { accuracy } \\
\text { Traditional } \\
\text { algorithm (\%) }\end{array}$ \\
\hline 1 & 95 & 82 \\
\hline 2 & 97 & 84 \\
\hline 3 & 96 & 83 \\
\hline 4 & 96 & 83 \\
\hline 5 & 94 & 81 \\
\hline 6 & 95 & 82 \\
\hline 7 & 97 & 81 \\
\hline 8 & 94 & 82 \\
\hline 9 & 95 & 83 \\
\hline 10 & 96 & \\
\hline
\end{tabular}

Based on the above results, and the experiment data are analyzed, the results show that the improved wavelet algorithm is better than the traditional method, the accuracy is higher than the traditional algorithm in image segmentation.

\section{Conclusion}

In this paper, the image segmentation is researched and optimized, the traditional algorithm is used for segmentation, the calculation is complex, and image segmentation accuracy rate is low, the clarity is not high, when the amount of information in the image segmentation is very large, the time consumption is big. Aiming at the above problems, an improved image optimization segmentation method is proposed based on wavelet algorithm. The image feature is obtained based on wavelet transform, the image characteristics can be reacted in local information, the advantage is obvious, the adjacent scale wavelet coefficients multiplicationmethod is used to remove the noise, and the wavelet algorithm and graph theory algorithm is fused to generate the minimum spanning tree, the accurate image segmentation is obtained with minimum spanning tree. The simulations show the method is applied in image segmentation, the precision is high, it has good application value in practice.

\section{References}

[1] Rao Yutai,Yang fan. Network Intrusion Stir the Network Instability Control Methods of the Research[J]. Bulletin of Science and Technology, 2014, 30(1):185-188.

[2] Guo Xiao-yan. Simulation and Analysis on Uncertain Attenuation Property of Underwater Acoustic Signal for Oil Field Pipe[J]. Computer Simulation, 2014,31(3):118-121.

[3] Guo Rong-yan, Hu Xue-hui. Study aboutLicense Plate Recognition Based on Back Propagation Neural Network[J]. Computer Simulation. 2010; 27(9): 299-301.

[4] Zheng Baodao, Wang Huaijie. Design of Remote Image Alarming System Based on ARM/GPRS. Computer Measurement \& Control. 2013; 21(1): 149-151, 159.

[5] Yang Zhi-Ming, Wang Xiao-Rong, Peng Jun. The Application of BP Neural Networks in Image Segmentation[J]. Computer Science. 2007; 30(3): 234-236. 